Bulletin of the AAS • Vol. 53, Issue 2

\title{
Peter O. Vandervoort (1935-2020)
}

\section{Louise Lerner ${ }^{1}$}

${ }^{1}$ University of Chicago

Published on: Jun 28, 2021

DOI: $10.3847 / 25 c 2 c f e b .45725 c f c$

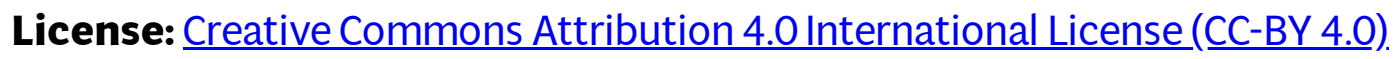




\section{Peter O. Vandervoort died on Friday the $11^{\text {th }}$ of December, 2020.}

Peter O. Vandervoort, an astrophysicist who was trained by legendary scientists at the University of Chicago and spent his career mentoring hundreds of students, colleagues and the public, died on Friday December 11, 2020. He was 85 years old.

Part of the University of Chicago community for nearly seven decades, Vandervoort, A.B. '54, S.B. '55, S.M. '56, Ph.D. '60, was remembered by colleagues as the resident historian of the Department of Astronomy \& Astrophysics and a lifelong champion for the University.

"In many ways, Peter was the embodiment of the history and culture in the astronomy department, but I also thought of him as embodying the ideals of the University," said Prof. Richard Kron, a longtime friend and colleague in the department. "He valued your opinion regardless of whether he disagreed, and he had the utmost respect for everyone as human beings and as participants in this academic world, which was really special."

Born April 25, 1935 in Detroit, Michigan, Vandervoort enrolled in the University of Chicago as an undergraduate in 1951. As his interest in physics grew, he spent time as a laboratory teaching assistant in a physics laboratory located under the West Stands of Stagg Field, near the site of the first controlled, self-sustaining nuclear chain reaction.

He started his Ph.D. surrounded by University of Chicago physicists who would later become legendary names in the field. His advisor was Subrahmanyan Chandrasekhar, who later won the 1983 Nobel Prize; 1963 Nobel laureate Maria Goeppert Mayer was on his Ph.D. committee; and Robert S. Mulliken, the 1966 Nobel laureate, served on his Ph.D. candidacy examination committee. 
Of being surrounded by these pioneering scholars, he told the Manhattan Project Voices: "You've been invited to Mount Olympus. What can I say?" He was also friends

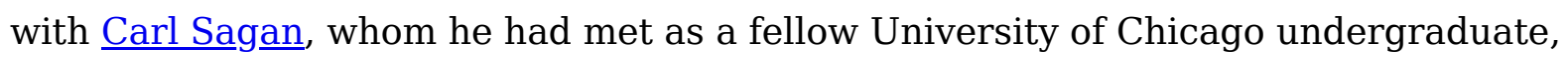
and they kept in touch for many years.

After receiving his Ph.D., he spent time at the National Radio Astronomy Observatory and the Princeton University Observatory. In 1961 he rejoined the University of Chicago as an assistant professor, and aside from a brief stint at the Leiden University Observatory in the Netherlands, he would remain at the University of Chicago for the rest of his life.

Vandervoort's area of specialty in astrophysics was stellar dynamics, the study of the movement of stars within galaxies. One of the oldest branches of theoretical astronomy, the field is heavily mathematical. Among other findings, Vandervoort helped fill in gaps in our understanding of the formation of spiral galaxies, calculated the orbits of stars inside these galaxies, and contributed to discussions of the role of chaos in stars and galaxies.

"It was clear that he was influenced by Chandrasekhar to think about elegant problems - places where nature would do something really interesting and there was a clever mathematical way of approaching how that process works," Kron said.

One of Vandervoort's greatest joys, however, was working with students. “That's a good way to see what an academic is all about, is to look at what kind of students they produce," Kron said.

One such former student, Ellen G. Zweibel, relayed her gratitude for Vandervoort in a letter nominating him for the Norman Maclean Faculty Award for teaching excellence. In 1971, she was a math student in the undergraduate College. She mused to Vandervoort about a possible idea she had; Vandervoort helped flesh it out and found a dataset to study the idea, then wrote it up as a paper.

"Although the idea would have been stillborn, and the paper would never have been written without Peter, he made me first author of the paper," wrote Zweibel, who is now a professor of astronomy at the University of Wisconsin-Madison. "Undergraduate research in astronomy is fairly common now, but at that time, it was rare. It was an emotional thunderbolt for me to realize that I could contribute to astronomical knowledge. He was a great mentor to me, and I have always thought that, in some ways, I owe my career to him." 
Friends and colleagues recollected that Vandervoort had a particular genius for matching students' skills and specialties to the areas that suited them best, and that his door was always open.

"Peter made an enormous contribution to the Department of Astronomy and Astrophysics, to the College, and to the University," said Prof. Angela V. Olinto, the Albert A. Michelson Distinguished Service Professor of Astronomy and Astrophysics and dean of the Physical Sciences Division. "He dedicated his life to scholarship, teaching, and to preserving the legacy of a pioneering and field-defining Chicago astronomy and astrophysics department, which will forever be in his debt."

Colleagues also remembered him as a gifted storyteller with a prodigious memory whether about encounters with Nobel laureates or the history and anatomy of telescope development at Yerkes Observatory.

"Peter was a source of inspiration for me. He was dedicated to the University and a constant reminder of the importance of our roles as educators and of the value of a liberal arts education," said John Carlstrom, the Subramanyan Chandrasekhar Distinguished Service Professor and chair of the astronomy department, and a colleague of Vandervoort's for decades. "Our paths often crossed at the end of the day, and when they did, I knew I would be late for dinner but have a wonderful story to tell. His stories about the rich history of the department brought out the best in all of us, especially for me as chair, to honor its legacy by always striving to further improve it and ourselves."

He served in multiple roles in the Department of Astronomy over the years, including as associate chair, the master of the Physical Sciences Collegiate Division and associate dean of the Physical Sciences Division. In particular, colleagues said, he constantly pushed to improve undergraduate instruction. He also led the planning and transition for two homes of the department - first an extensive renovation to the former Astronomy \& Astrophysics building and later the design of its current home in the William Eckhardt Research Center. 


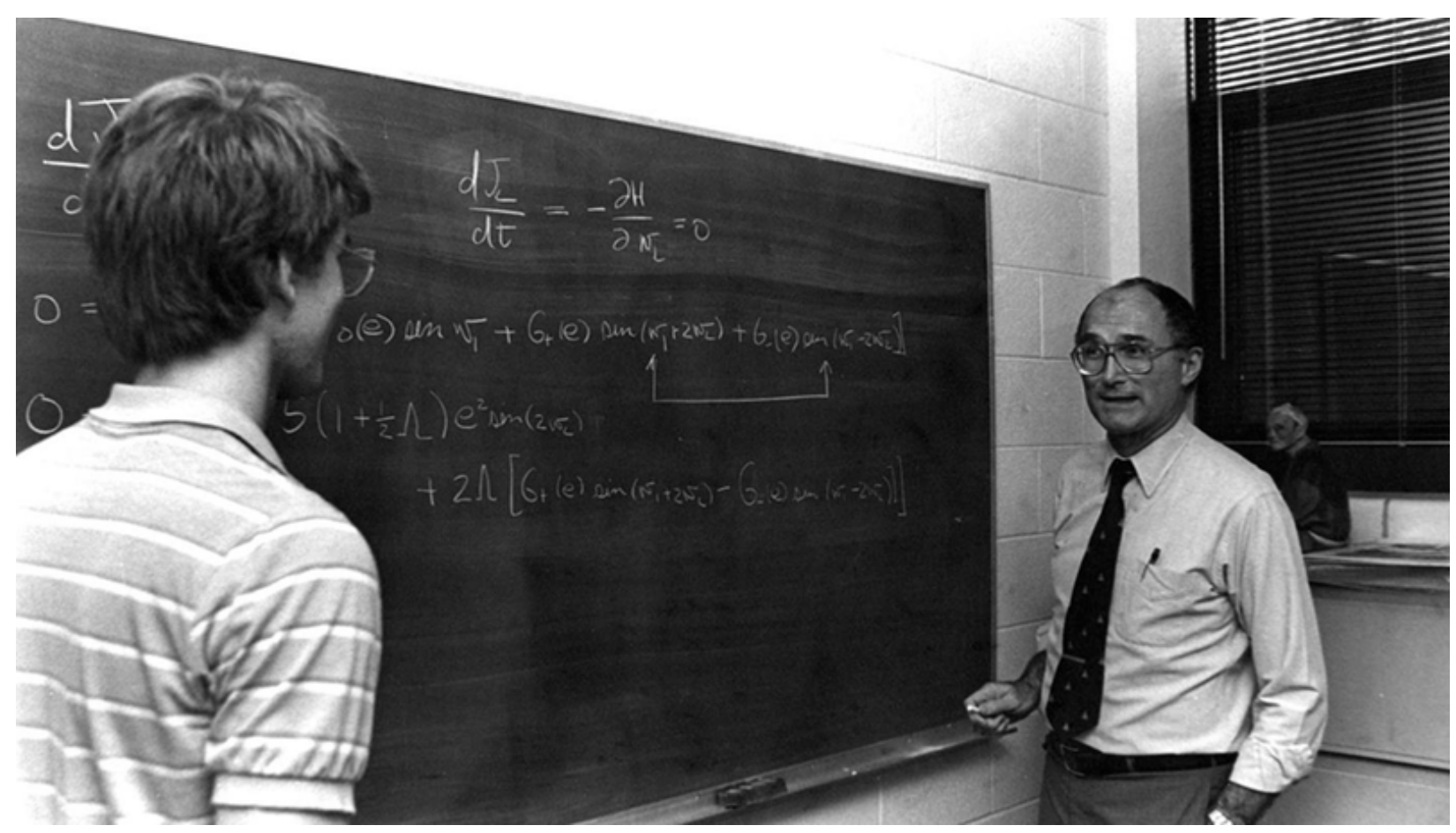

Vandervoort working with student Eric Sather in 1984 (photo by Richard Dreiser, courtesy Hanna Holborn Gray Special Collections Research Center)

"In many ways, he was an institution here," said Prof. Emeritus Michael Turner, another longtime colleague. "He was a person of enormous integrity. We are a much better institution for Peter having been here, and the department, the College and the University will not be the same without him."

As the keeper of history for the Department of Astronomy, it was natural for Vandervoort to serve with the Adler Planetarium in Chicago. Vandervoort was first appointed a trustee in 1974; in 2004, he was made a Life Trustee. In particular, he served for many years on the committee that oversees the preservation of astronomical artifacts.

"What we do at Adler is tell stories - about the enduring connection between the sky, the universe and humans - so having Peter as a colleague who was also adept at that kind of storytelling was a true gift," said Andrew Johnston, vice president of museum experience and collections at the Adler Planetarium. "He could give us the grand sweep of the history of astronomy, but also the profound ways that the science has advanced right here in Chicago - and he was witness."

Vandervoort was a member of the American Astronomical Society, the International Astronomical Union, the American Physical Society, the Royal Astronomy Society, and the American Association for the Advancement of Science. 
He is survived by his wife of 64 years, Frances (S.B. '57, S.M. '65), sons William and Dirk, and granddaughter Abigail.

Adapted from the obituary written for the University of Chicago News by Louise Lerner, Dec 17, 2020. 